\title{
Influence of Nitrogen Deficiency on Ureide Mediated Heavy Metal Stress Resistance in Arabidopsis thaliana
}

\author{
Talisa K. Silzer* \& Christopher D. Todd
}

\begin{abstract}
The relationship between nitrogen availability and presence of heavy metal stress appear to affect development and growth of Arabidopsis thaliana plants. In this work, Arabidopsis thaliana seedlings were exposed to media containing varying concentrations of nitrogen in form of $\mathrm{NH}_{4} \mathrm{NO}_{3}$ or $\mathrm{KNO}_{3}$ and cadmium in the form of $\mathrm{CdCl}_{2}$. When exposed to increasing cadmium stress, aln mutant lines, lacking a functional allantoinase enzyme, displayed enhanced growth and development when compared to wild type, most likely due to their high allantoin content. Increasing nitrogen concentrations appeared to decrease the amount of senescing tissue caused by exposure to cadmium stress. Growth appeared to be slightly increased in both wild type and aln mutant lines grown in media containing $\mathrm{KNO}_{3}$ as the nitrogen source, thereby supporting the importance of nitrogen source on plant growth.
\end{abstract}

Keywords: nitrogen deficiency, arabidopis thaliana, heavy metals, cadmium stress

\section{Introduction}

The growth and development of a plant primarily depends on the availability of a few key macronutrients such as carbon, oxygen and hydrogen. In general, plants are able to obtain these macronutrients from the atmosphere and surrounding water sources. However, an essential and potentially restrictive macronutrient for a plant is nitrogen (Vidal and Gutierrez, 2008). Nitrogen holds great significance in plants, as it is a major element of chlorophyll, the molecule responsible for producing energy for the plant via absorption of sunlight (SanchezZabala et al., 2015). In addition, nitrogen is also a major building block for nucleic acids and amino acids, and plays key roles in controlling gene expression (Vidal and Gutierrez, 2008).

The model plant Arabidopsis thaliana ( $A$. thaliana) uses the purine oxidation pathway, otherwise referred to as the ureide metabolic pathway, to degrade purine or pyrimidine bases into functional nitrogen sources such as ammonium (Werner and Witte, 2011; Yang and Han, 2004). This pathway consists of a series of enzymatic reactions involving 10 different intermediates (Werner et al., 2013). Many of the intermediates associated with this pathway, specifically allantoin, appear to be nitrogen-rich and therefore serve an important role in nitrogen assimilation, transport and storage throughout the shoots and leaves of the plant (Yang and Han, 2004). In particular, the AtALN gene

*Department of Biology, College of Arts and Science, University of Saskatchewan, Saskatoon, SK, Canada

Correspondence: talisasilzer@gmail.com

University of Saskatchewan Undergraduate Research Journal

Volume 4, Issue 1, 2017 
encoding for the allantoinase enzyme is responsible for synthesizing allantoate from allantoin. When mutated, the AtALN gene results in defective allantoinase, thereby triggering elevated allantoin levels that may be associated with increased tolerance to abiotic stresses (Watanabe et al., 2014). Watanabe et al. (2014) have proposed that increased allantoin levels may activate abscisic acid (ABA) metabolism, which in turn upregulates various drought and osmotic stress response genes.

Although abiotic stress responses in A. thaliana loss of function mutants such as those of xanthine dehydrogenase $(X D H)$, allantoinase $(A L N)$ and allantoate aminohydrolase $(A A H)$ have been studied, little work has been done on the effect of cadmium as an abiotic stress and the interaction that cadmium may have with critical plant nutrients such as nitrogen. Cadmium is a toxic heavy metal that is most commonly found in environments subject to anthropogenic pressure (Sanita di Toppi and Gabbrielli, 1999). It is rapidly taken up by plant roots and accumulated in plant tissue altering the rate of photosynthesis, uptake of nutrients and water use efficiency (Gill and Tuteja, 2011; Perez-Chaca et al., 2014). Exposure to cadmium also results in increased accumulation of hydrogen peroxide and reactive oxygen species (ROS) that serve as regulators of programmed cell death (Maksymiec and Krupa, 2006; Gechev et al., 2005). Concentrations of ROS are often dose and time dependent, therefore higher levels of cadmium result in increased death of plant tissue (Perez-Chaca et al., 2014).

The purpose of this study was to analyze the interaction between varying concentrations of environmental nitrogen and cadmium, and determine how this interaction may affect the growth of wild type (WT) and mutant aln A. thaliana seedlings. Nourimand and Todd (2016) have observed enhanced growth of aln-3 mutant lines in both agar and soil environments subjected to cadmium. In addition, the increased tolerance of aln mutants to other abiotic stresses such as high osmoticum and enhanced drought has been attributed to their high levels of allantoin (Watanabe et al., 2014). It has also been noted that increased nitrogen levels decrease the amount of cadmium uptake by plant roots (Konotop et al., 2012). Based on the available literature, aln mutants were hypothesized to display better growth and enhanced tolerance to cadmium stress when compared to wild type plants, due to the characteristic allantoin accumulation observed in aln mutant lines.

\section{Materials and Methods}

\section{Plants and Growth Conditions}

A. thaliana wild type (WT) and mutant aln lines were used in this work. The SALK T-DNA insertion line, aln was obtained from the Arabidopsis Biological Resource Center at Ohio State University. Surface sterilized seeds from both WT and transgenic plants were grown on $9 \mathrm{~cm}$ plates containing Murashige and Skoog (MS) basal salts, $0.5 \%$ sucrose, $1.2 \%$ agar and varying concentrations of both nitrogen and cadmium. Nitrogen was provided in the form of ammonium nitrate $\left(\mathrm{NH}_{4} \mathrm{NO}_{3}\right)$ and potassium nitrate $\left(\mathrm{KNO}_{3}\right)$ in concentrations of o $\mathrm{mM}$, $11.25 \mathrm{mM}$ and $22.5 \mathrm{mM}$, while cadmium was provided in the form of cadmium chloride $\left(\mathrm{CdCl}_{2}\right)$ in concentrations of o $\mu \mathrm{M}, 50 \mu \mathrm{M}$ and $100 \mu \mathrm{M}$. Following incubation at $4^{\circ} \mathrm{C}$ for two days, plates were placed in a growth chamber with a light intensity of $70 \mu \mathrm{mol}$ photons $\mathrm{m}^{-2} \mathrm{~s}^{-1}$ for fourteen days at $22 \circ \mathrm{C}$ with a photoperiod of sixteen hours as described by Nourimand and Todd (2016).

\section{Phenotypic Comparisons and Average Root Length Measurement}

A. thaliana plants from each treatment were compared visually based on size, color, root length and root branching. Roots of $A$. thaliana plants were measured using Image J software version 1.46r. Average root length was measured and recorded in centimeters.

\section{High Performance Liquid Chromatography}

Following the protocol described by Nourimand and Todd (2016), approximately $60 \mathrm{mg}$ of fresh tissue was sampled and homogenized with distilled water using a mortar and pestle. Following centrifugation at $2^{\circ} \mathrm{C}$ at maximum speed (14,000 rpm) for 25 minutes, supernatant was removed and dispensed through a $3 \mathrm{~mL}$ syringe with a $13 \mathrm{~mm}$ filter (VWR International) into a 1.5 $\mathrm{mL}$ HPLC vial. Samples were run in an Agilent Technologies 1200 series HPLC with an Aminex HPX-87H Ion Exclusion column, using diluted sulfuric acid $\left(\mathrm{H}_{2} \mathrm{SO}_{4}\right)$ solution consisting of $0.0025 \mathrm{~N} \mathrm{H}_{2} \mathrm{SO}_{4}$ and distilled $\mathrm{H}_{2} \mathrm{O}$ for 55 minutes. Concentrations of allantoin within the samples were compared to standard curves generated from pure allantoin (Sigma-Aldrich). 
Nitrogen Concentration (mM)

0



Figure 1: WT and aln Arabidopsis plants treated with varying concentrations of nitrogen (supplied as $\mathrm{NH}_{4} \mathrm{NO}_{3}$ ) in the presence of o $\mu \mathrm{M}$ cadmium. Representative images are shown.

\section{Nitrogen Concentration (mM)}

O

11.2

22.5

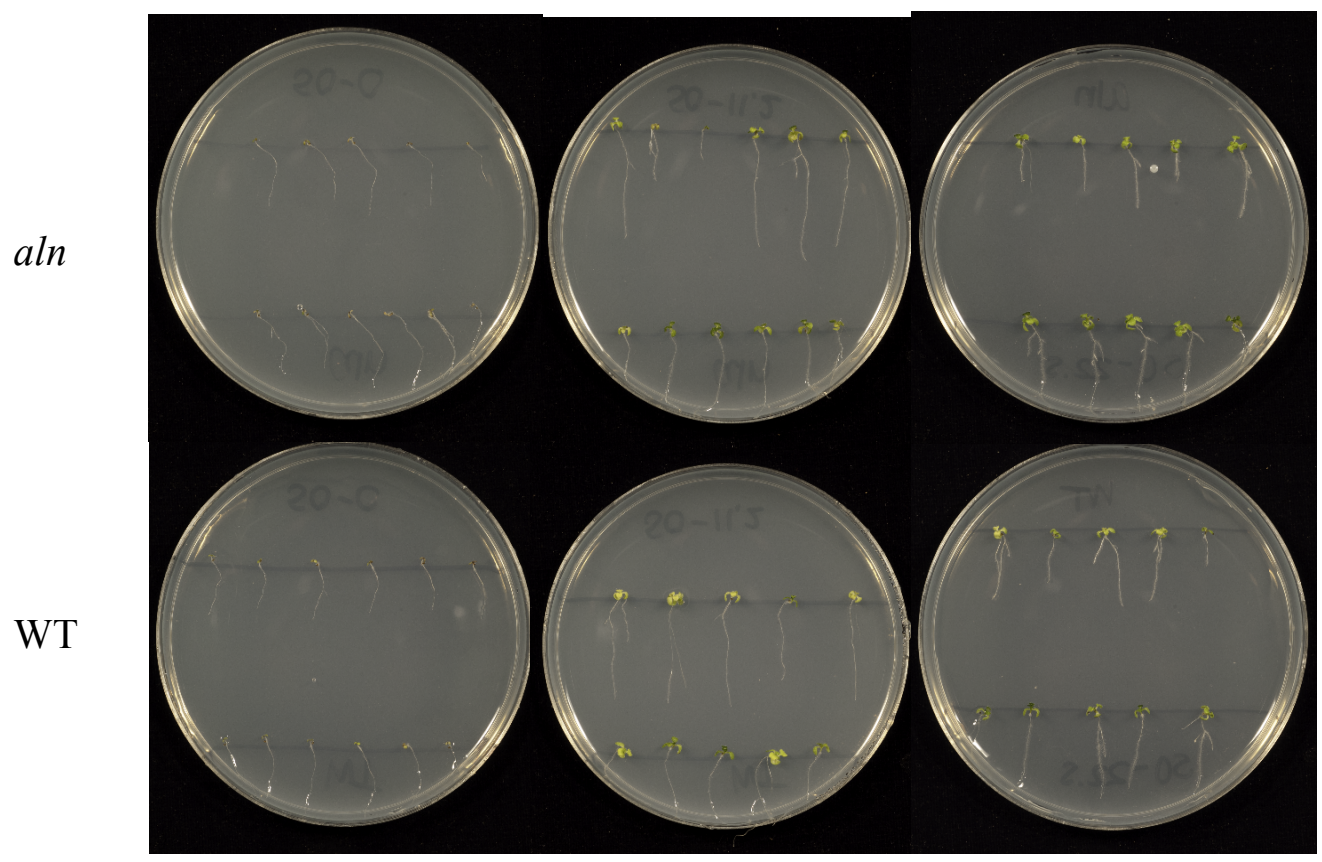

Figure 2: WT and aln Arabidopsis plants treated with varying concentrations of nitrogen (supplied as $\mathrm{NH}_{4} \mathrm{NO}_{3}$ ) in the presence of $50 \mu \mathrm{M}$ cadmium (supplied as $\mathrm{CdCl}_{2}$ ). Representative images are shown 


\section{Statistical Analysis}

Data shown in figures are the means of three independent replicates, with exception to the seedlings grown in $\mathrm{KNO}_{3}$, which comprised only two independent replicates. Both means and \pm SEM values were calculated using Microsoft Excel.

\section{Results}

Arabidopsis WT and aln plants were plated on media containing varying concentrations of nitrogen at o $\mathrm{mM}$, $11.2 \mathrm{mM}$ or $22.5 \mathrm{mM}$ and $o \mu \mathrm{M}, 50 \mu \mathrm{M}$ or $100 \mu \mathrm{M}$ of cadmium. Following a 14-day growth period, plants were compared visually based on size, tissue color and root length. When grown in the absence of cadmium, both WT and aln plants appeared to have better growth. However, when grown in the absence of nitrogen, both WT and aln mutants showed minimal growth. Furthermore, when treated with $11.2 \mathrm{mM}$ and $22.5 \mathrm{mM}$ nitrogen, aln mutants were observed to have slightly better growth with increased root branching and lack of yellowing tissue, a hallmark of senescence (Figure 1).

Moreover, when grown in the presence of $50 \mu \mathrm{M}$ cadmium, WT and aln mutants appeared to have very similar growth and color. In the absence of nitrogen, minimal growth was observed for both WT and aln. However, when treated with $11.2 \mathrm{mM}$ and $22.5 \mathrm{mM}$ nitrogen, aln mutants displayed increased root branching in comparison to WT (Figure 2).

Furthermore, when grown in the presence of 100 $\mu \mathrm{M}$ cadmium, drastic differences were observed in growth and health of WT and aln plants. In the absence of nitrogen, both WT and aln seeds were incapable of germinating, thereby producing no growth (Figure 3). However, when treated with $11.2 \mathrm{mM}$ and $22.5 \mathrm{mM}$ nitrogen, aln mutants displayed significantly better growth and very little tissue yellowing in comparison to WT (Figure 3).

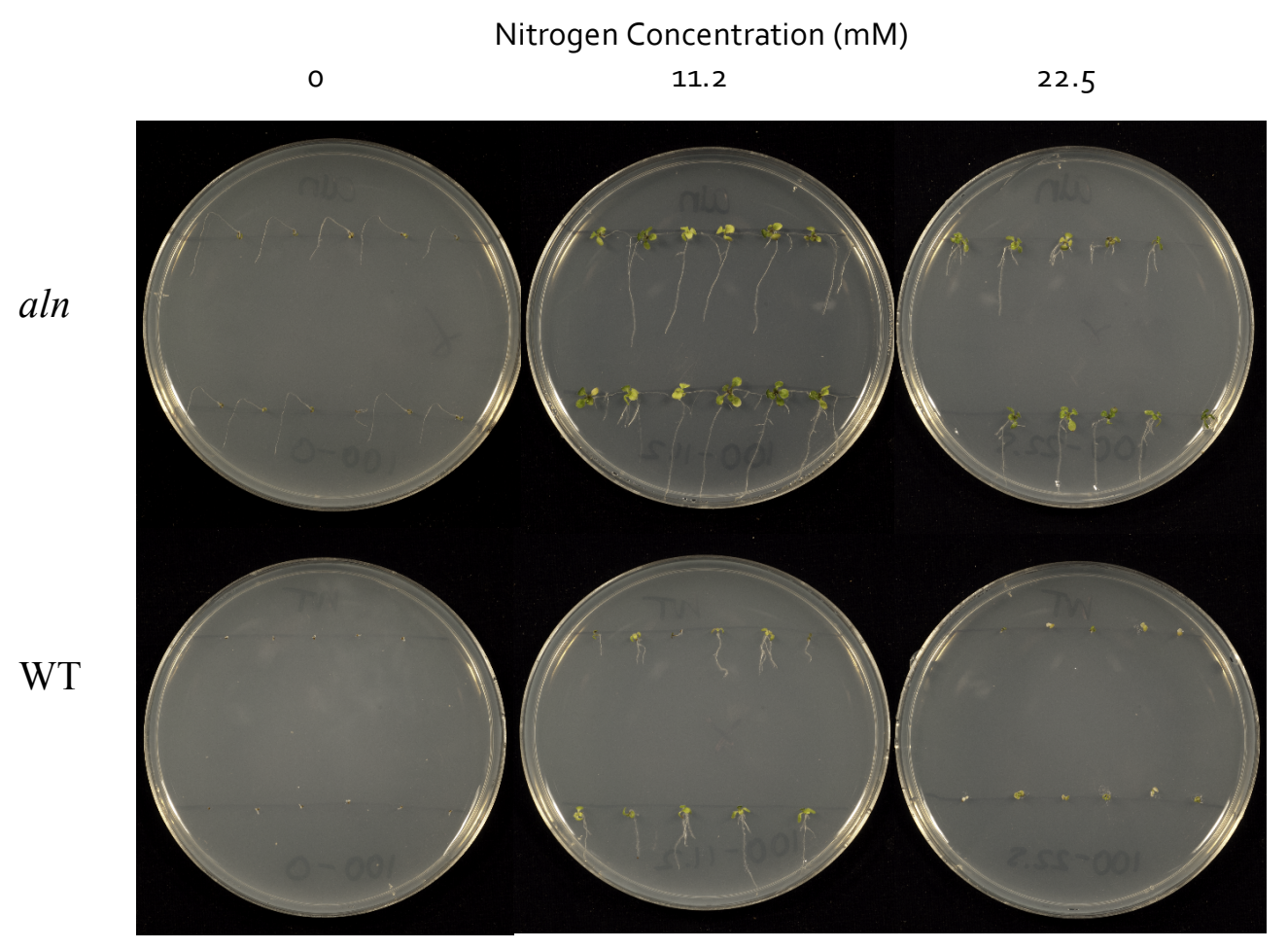

Figure 3: WT and aln Arabidopsis plants treated with varying concentrations of nitrogen (supplied as $\mathrm{NH}_{4} \mathrm{NO}_{3}$ ) in the presence of $50 \mu \mathrm{M}$ cadmium (supplied as $\mathrm{CdCl}_{2}$ ). Representative images are shown 
Lastly, when comparing growth of WT and aln plants grown at $50 \mu \mathrm{M}$ cadmium and $11.2 \mathrm{mM}$ and $22.5 \mathrm{mM}$ nitrogen in the form of $\mathrm{KNO}_{3}$, very similar growth was observed with distinct differences only found in root length. Both WT and aln appeared to be yellow in color.

Average root length was quantified using Image J software, allowing for numerical comparisons between WT and aln Arabidopsis plants treated with varying concentrations of nitrogen and cadmium. In general, root lengths between the two genotypes appeared to be more distinct with increasing cadmium concentration.

When grown in media lacking cadmium, WT and aln plants were observed to have very similar average root lengths. When grown in the absence of nitrogen as well, both WT and aln showed similar average root lengths of approximately $0.5 \mathrm{~cm}$ (Figure $4 \mathrm{~A}$ ). However, when treated with $11.2 \mathrm{mM}$ nitrogen, WT plants appeared to have a $20 \%$ higher average root length in comparison to aln (Figure $4 \mathrm{~A}$ ). Conversely, when treated with 22.5 $\mathrm{mM}$ of nitrogen, aln plants appeared to have a $22 \%$ higher average root length in comparison to WT (Figure 4A).

In most cases, when grown in media containing $50 \mu \mathrm{M}$ cadmium, aln mutants appeared to have longer average root length than WT. When grown in the absence of nitrogen, aln plants appeared to have $56 \%$ higher average root length than WT, yet WT plants appeared to have $20 \%$ higher average root length than aln when treated with $11.2 \mathrm{mM}$ nitrogen (Figure $4 \mathrm{~B}$ ). Furthermore, when treated with $22.5 \mathrm{mM}$ nitrogen, aln mutants displayed a $62 \%$ increase in root length in comparison to WT (Figure 4B).

When grown in media containing $100 \mu \mathrm{M}$ cadmium, drastic differences in average root length were noticed between the two genotypes. When grown in media lacking nitrogen, WT plants showed no germination and therefore no root growth, while aln developed roots of approximately $1 \mathrm{~cm}$ in length (Figure $\left.{ }_{4} \mathrm{C}\right)$. In addition, when treated with $11.2 \mathrm{mM}$ nitrogen, aln plants appear to have a $173 \%$ increase in root length when compared to WT (Figure ${ }_{4} \mathrm{C}$ ). Furthermore, aln mutants appeared to have an approximate $676 \%$ increase in root length in comparison to WT when treated with $22.5 \mathrm{mM}$ nitrogen (Figure ${ }_{4} \mathrm{C}$ ).

In order to analyze the effect of nitrogen source on growth of WT and aln mutants under cadmium stress, average root length was also quantified for Arabidopsis plants grown in media containing $\mathrm{KNO}_{3}$ as the nitrogen source. Overall, average root length appeared to be longer for both WT and aln plants exposed to $50 \mu \mathrm{M}$ cadmium when treated with $\mathrm{KNO}_{3}$ instead of $\mathrm{NH}_{4} \mathrm{NO}_{3}$. When treated with $11.2 \mathrm{mM}$ nitrogen in the form of $\mathrm{KNO}_{3}$,

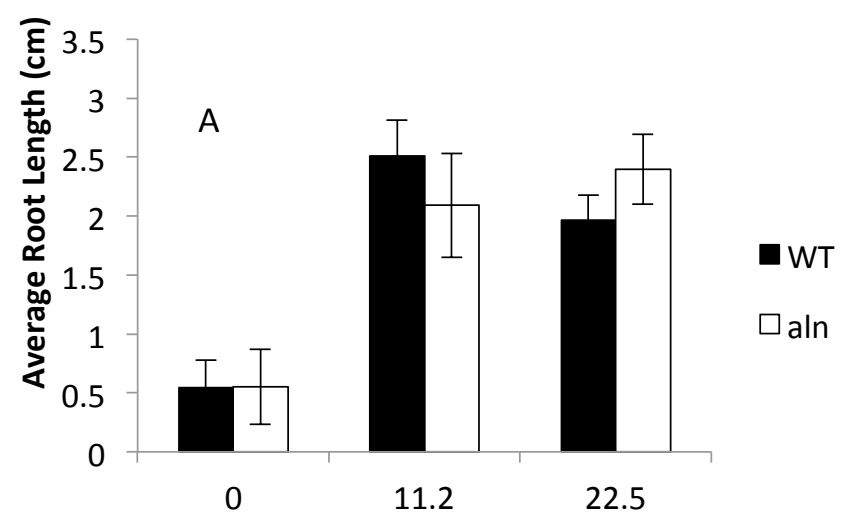

Nitrogen Concentrations (mM)

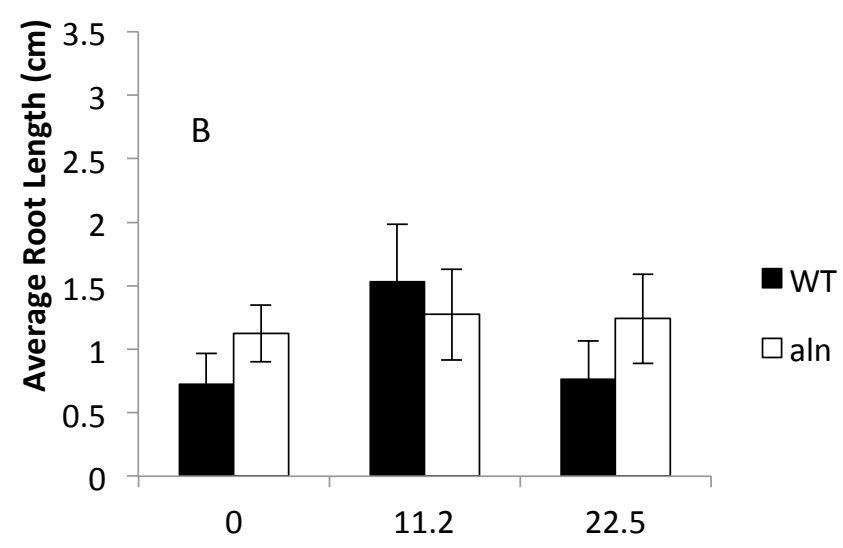

Nitrogen Concentrations (mM)



Figure 4: Average root length for WT and aln Arabidopsis plants treated with varying concentrations of nitrogen (supplied as $\mathrm{NH}_{4} \mathrm{NO}_{3}$ ) in the presence of A. 0, B. 50, C. $100 \mu \mathrm{M}$ cadmium (supplied as $\mathrm{CdCl}_{2}$ ). Data shown represents the average root length for three independent replicates of WT (black bars) and aln (white bars). Absence of bar indicates no allantoin detected. Error bars represent \pm SEM. 


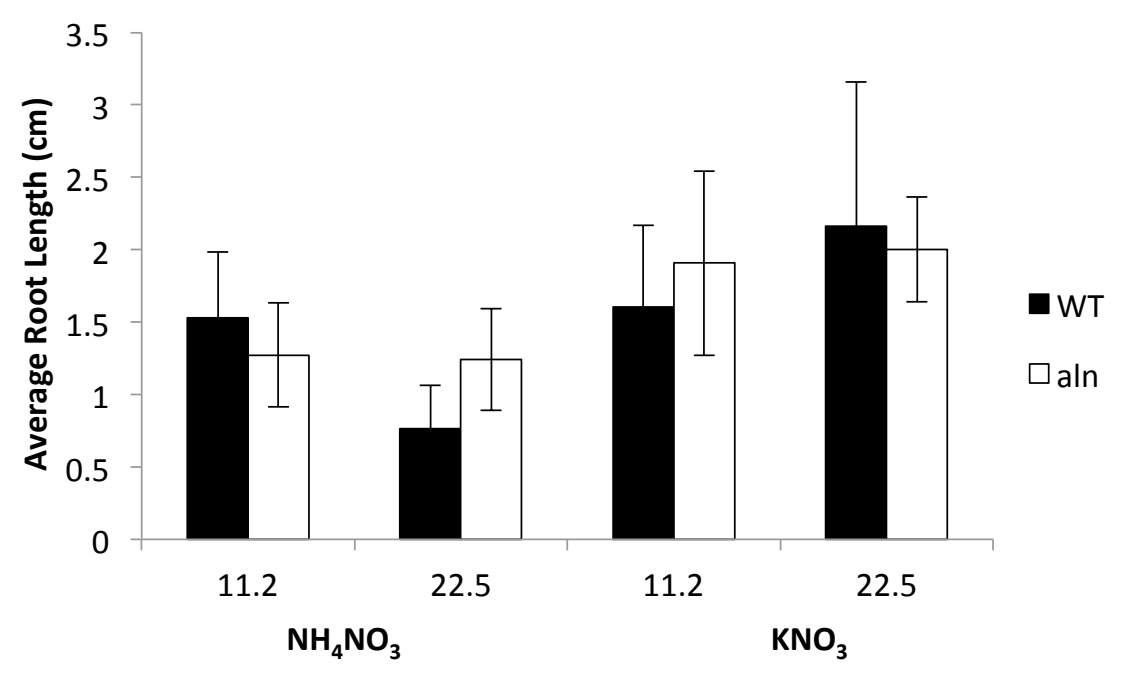

Figure 5: Average root length for WT and aln Arabidopsis plants treated with varying concentrations of nitrogen (supplied as $\mathrm{NH}_{4} \mathrm{NO}_{3}$ or $\mathrm{KNO}_{3}$ ) in the presence of $50 \mu \mathrm{M}$ cadmium (supplied as $\mathrm{CdCl}_{2}$ ). Data shown represents the average root length for three independent replicates of WT (black bars) and aln (white bars). Error bars represent \pm SEM.

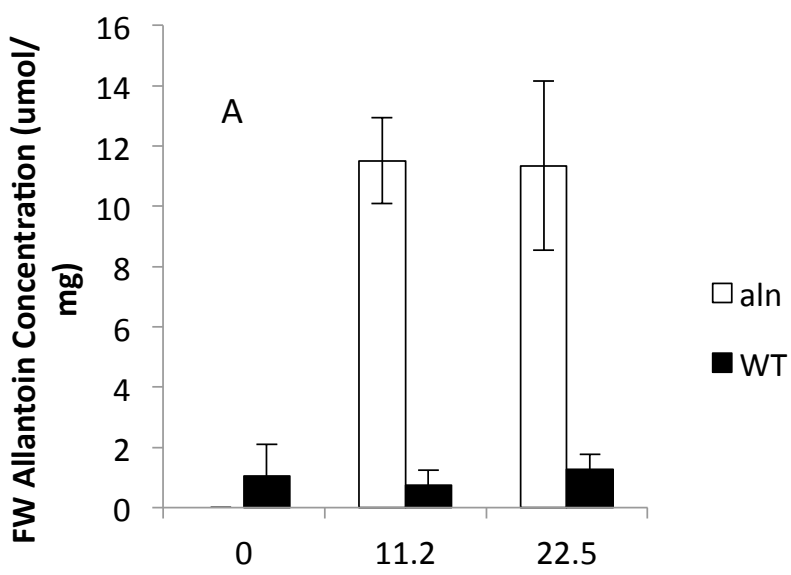

Nitrogen Concentration (mM)
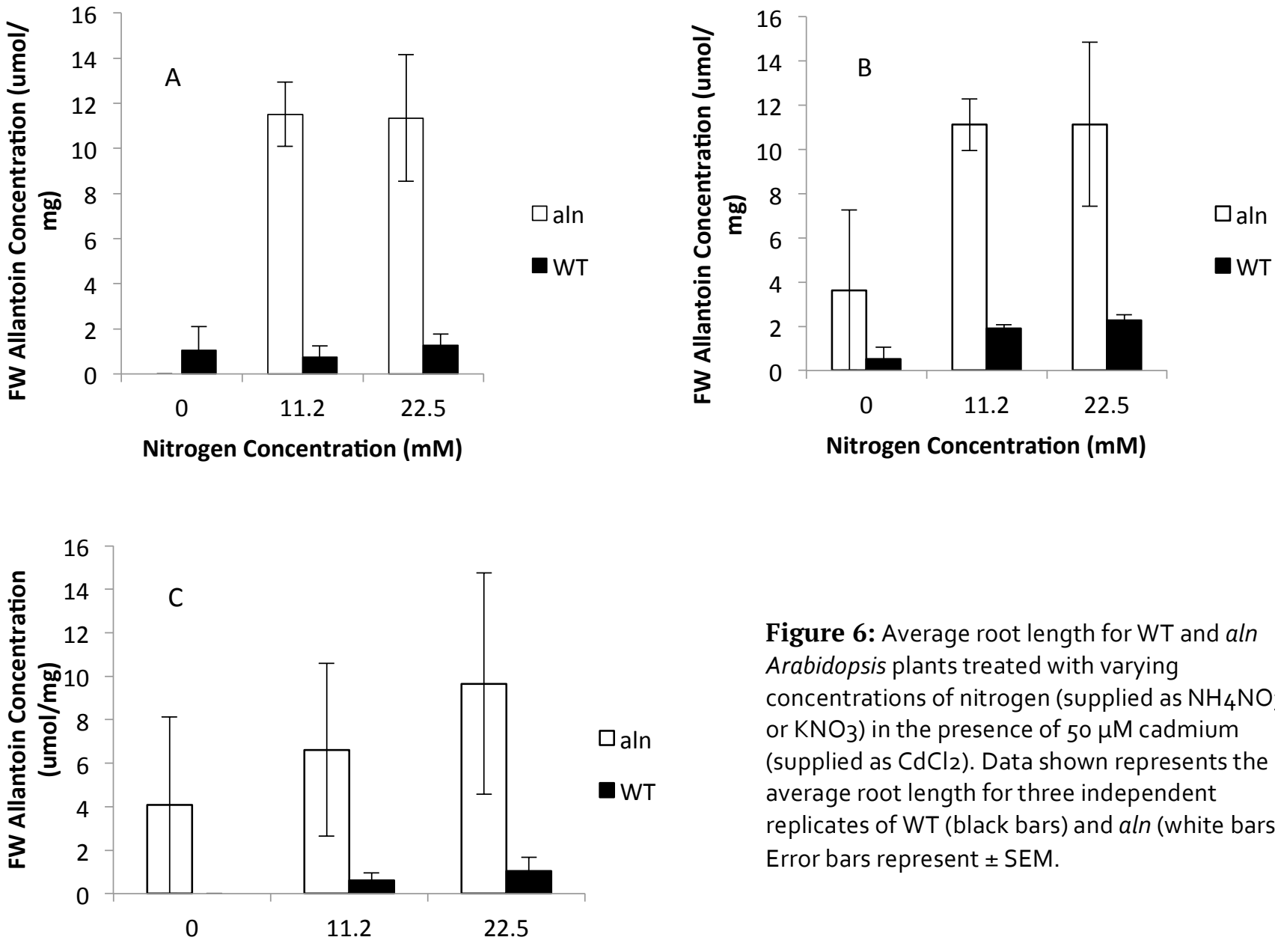

Figure 6: Average root length for WT and aln Arabidopsis plants treated with varying concentrations of nitrogen (supplied as $\mathrm{NH}_{4} \mathrm{NO}_{3}$ or $\mathrm{KNO}_{3}$ ) in the presence of $50 \mu \mathrm{M}$ cadmium (supplied as $\mathrm{CdCl}_{2}$ ). Data shown represents the average root length for three independent replicates of WT (black bars) and aln (white bars). Error bars represent \pm SEM.

Nitrogen Concentration (mM) 


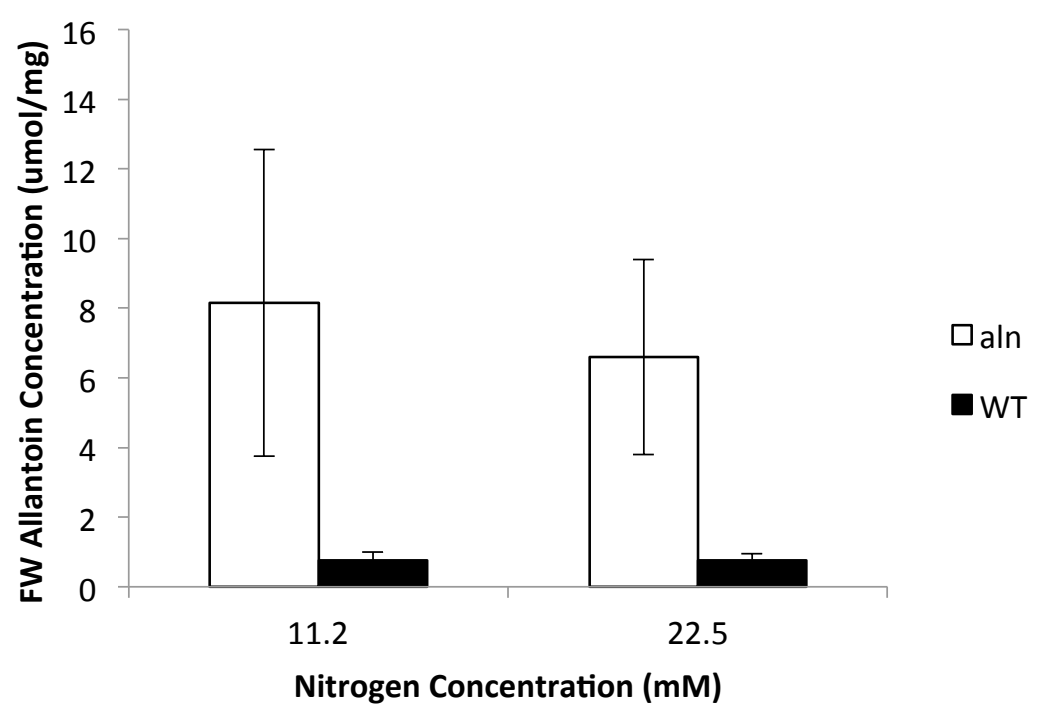

Figure 7: Average concentration of allantoin in fresh weight (FW) samples of WT and aln Arabidopsis plants treated with varying concentrations of nitrogen (supplied as $\mathrm{KNO}_{3}$ ) in the presence of 50 $\mu \mathrm{M}$ cadmium (supplied as $\mathrm{CdCl}_{2}$ ). Data shown represents the average allantoin concentration for two independent replicates for WT (black bars) and aln (white bars). Error bars represent \pm SEM. aln mutants showed a $19 \%$ increase in average root length compared to WT (Figure 5). However, when treated with $22.5 \mathrm{mM}$ nitrogen in the form of $\mathrm{KNO}_{3}$, WT plants displayed an $8 \%$ increase in average root length in comparison to aln mutants (Figure 5). Similarly, WT plants appeared to have $20 \%$ higher average root length than aln when treated with $11.2 \mathrm{mM}$ of nitrogen in the form of $\mathrm{NH}_{4} \mathrm{NO}_{3}$ (Figure 5). However, aln mutants appeared to have a $62 \%$ longer average root length in comparison to WT (Figure 5).

Next, allantoin concentration was quantified for WT and aln samples treated with varying concentrations of $\mathrm{CdCl}_{2}, \mathrm{NH}_{4} \mathrm{NO}_{3}$ and $\mathrm{KNO}_{31}$ using HPLC. As predicted, allantoin concentrations appeared to be significantly higher in aln mutant lines. When grown in media lacking cadmium and nitrogen, aln mutants had zero allantoin content, while WT had a minimal average allantoin concentration of approximately $1 \mu \mathrm{mol} / \mathrm{mg}$ (Figure 6A). Furthermore, when treated with $11.2 \mathrm{mM}$ and $22.5 \mathrm{mM}$ nitrogen, aln mutants had allantoin content 15 fold and 8.9 fold higher than WT respectively (Figure 6A).

Similar allantoin concentrations were also observed in WT and aln plants subjected to $50 \mu \mathrm{M}$ cadmium. When grown in media lacking nitrogen, aln mutants appeared to have a 7 -fold increase in allantoin content in comparison to WT (Figure 6B). In addition, plants grown in media containing $11.2 \mathrm{mM}$ and $22.5 \mathrm{mM}$ of nitrogen, aln mutants appeared to have respective 6fold and 5 -fold increases in allantoin concentrations in comparison to WT (Figure 6B).

Lastly, allantoin concentrations for plants exposed to $100 \mu \mathrm{M}$ cadmium appeared to be slightly decreased in aln mutants but still significantly higher than WT plants. When grown in media lacking nitrogen, zero allantoin was observed in WT, while aln mutants were observed to have an allantoin concentration of approximately $4 \mu \mathrm{mol} / \mathrm{mg}$ (Figure $6 \mathrm{C}$ ). In addition, when treated with $11.2 \mathrm{mM}$ and $22.5 \mathrm{mM}$ of nitrogen, aln mutants were observed to have allantoin concentrations 10.7 and 9.2-fold higher than WT respectively (Figure 6C).

Allantoin content was also quantified for WT and aln plants grown in media containing $50 \mu \mathrm{M}$ of cadmium and $11.2 \mathrm{mM}$ and $22.5 \mathrm{mM}$ of nitrogen in the form of $\mathrm{KNO}_{3}$. In plants treated with $11.2 \mathrm{mM}$ of nitrogen, aln plants were observed to have allantoin concentrations 10.8-fold higher than WT plants (Figure 7). Furthermore, when treated with $22.5 \mathrm{mM}$ of nitrogen, aln plants had allantoin concentrations 8.7-fold higher than WT (Figure 7)

\section{Discussion}

In this study, phenotypic comparisons and HPLC analysis provided evidence to suggest that aln mutant Arabidopsis lines display superior growth over WT when exposed to abiotic stress in the form of cadmium. Since allantoin has been known to increase abiotic stress tolerance in plants by activating $A B A$ stress response genes such as RD29A, $\mathrm{RD}_{29} \mathrm{~B}$ and RD26, this may explain why the aln mutants displayed increased growth in comparison to WT (Watanabe et al., 2014). When grown in media lacking cadmium, aln mutant lines and WT plants were observed to have differences in color, but relatively similar average root lengths. Furthermore, with the addition of nitrogen 
to the media, both WT and aln mutants appeared to have better growth, which was expected in the absence of stress. Although WT plants appeared to have a slightly higher average root length than aln mutants when treated with o $\mu \mathrm{M}$ cadmium and $11.2 \mathrm{mM}$ nitrogen, aln mutants displayed better growth with increased root branching and less tissue yellowing in comparison to WT when treated with $22.5 \mathrm{mM}$ nitrogen. Furthermore, since allantoin concentrations were found to be significantly higher in aln mutants, it may be that high allantoin concentrations allow for better growth even in the absence of stress (Watanabe et al., 2014).

When grown in media containing $50 \mu \mathrm{M}$ cadmium, WT and aln plants displayed very similar growth. In the presence of $11.2 \mathrm{mM}$ and $22.5 \mathrm{mM}$ nitrogen, aln mutants were observed to have similar color but slightly more root branching in comparison WT. Although aln mutants displayed longer average root length when exposed to $22.5 \mathrm{mM}$ nitrogen, WT plants displayed slightly longer average root length when exposed to $11.2 \mathrm{mM}$ nitrogen. This is not surprising, as similar increases in root length have also been observed in WT Glycine Max plants exposed to cadmium and medium doses of nitrogen (Konotop et al., 2012). This slight increase in root length observed in WT may be due to low replicate number. However, it may be possible that at $50 \mu \mathrm{M}$ cadmium and $11.2 \mathrm{mM}$ nitrogen, WT plants act as they would in a soil environment and are able to develop longer roots to aid in pursuing nutrients that would allow for survival.

In the presence of $100 \mu \mathrm{M}$ cadmium, WT and aln plants displayed significant differences in growth. In the absence of nitrogen, both WT and aln seedlings were incapable of germinating. This result was expected due to the presence of stress and lack of nutrients. aln mutants also displayed considerably longer average root length and better growth at $11.2 \mathrm{mM}$ and $22.5 \mathrm{mM}$ nitrogen in comparison to WT. This increase in root length and enhanced growth correlates with the high levels of allantoin observed in aln mutants. Furthermore, WT plants were observed to have very minimal allantoin content, which may explain why they displayed little growth under high abiotic stress conditions. As observed in previous studies, allantoin accumulation may lead to activation of ABA stress response genes (Watanabe et al., 2014). Increased expression of ABA stress response genes may further explain why aln mutants display better growth than WT, specifically when exposed to considerable abiotic stress.

The results suggest that there is an interaction between nitrogen and abiotic stresses such as cadmium and that this interaction aids in determining the amount of plant growth for both WT and aln. However, this interaction may be more complex than originally thought. Analysis of WT and aln plants exposed to $50 \mu \mathrm{M}$ cadmium and $11.2 \mathrm{mM}$ and $22.5 \mathrm{mM}$ nitrogen in the form of $\mathrm{KNO}_{3}$ confirms that the nitrogen source may also play a key role in determining plant growth in the presence of abiotic stress. When comparing WT plant growth, we see increased root length in those treated with $\mathrm{KNO}_{3}$ as the nitrogen source. Given the limited number of replicates used in this study, future studies may focus on reproducing these findings using a larger sample size to determine whether the associations between nitrogen presence and/or cadmium stress, and plant growth are significant.

\section{Conclusion}

The purpose of this study was to analyze the interaction between cadmium and nitrogen and determine how this interaction may affect plant growth. It was hypothesized that aln mutant Arabidopsis plants would display better growth in comparison to WT when exposed to cadmium, due to their characteristic accumulation of allantoin. In addition, it was also predicted that both WT and aln plants grown in nitrogen containing media would show enhanced growth in comparison to plants grown in the absence of nitrogen. In general, results from this study have supported the original hypothesis. Under cadmium stress, aln mutant lines appeared to display better growth with longer and more branched roots and little senescing tissue, particularly in media containing nitrogen. Increased growth and resistance to cadmium stress was most likely attributed to the high allantoin concentrations observed in aln mutants. Furthermore, WT plants appeared to have better growth under cadmium stress when grown in the presence of nitrogen. Lastly, it appears that the interaction between cadmium and nitrogen is also dependent on the nitrogen source. Although only slight differences were noticed between the phenotypes of plants treated with $\mathrm{NH}_{4} \mathrm{NO}_{3}$ and $\mathrm{KNO}_{3 \prime}$ it may be beneficial to analyze the interaction between cadmium and other nitrogen sources to further understand the affects that this interaction may have on plant growth. 


\section{Acknowledgements}

I would like to thank Maryam Nourimand and my fellow lab mates for their assistance and guidance in the lab. Furthermore, I would like to thank photography technician, Marlynn Mierau for his dedication and time. Lastly, I would like to thank the University of Saskatchewan Biology department for providing the proper equipment and facilities that this project demanded. This project was supported by NSERC Discovery Grant 327190 to Christopher Todd.

\section{References}

Di Toppi, L.S. and R. Gabbrielli, Response to cadmium in higher plants. Environmental and Experimental Botany, 1999. 41(2): p. 105-130.

Gechev, T., I. Minkov, and J. Hille, Hydrogen peroxideinduced cell death in Arabidopsis: transcriptional and mutant analysis reveals a role of an oxoglutarate-dependent dioxygenase gene in the cell death process. IUBMB life, 2005. 57(3): p. 181.

Gill, S.S. and N. Tuteja, Cadmium stress tolerance in crop plants: Probing the role of sulfur. Plant Signaling \& Behavior, 2011. 6(2): p. 215.

Konotop, Y., et al., Application of nitrogen nutrition for improving tolerance of soybean seedlings to cadmium. Environ Exp Biol, 2012. 10: p. 139-144.

Maksymiec, W. and Z. Krupa, The effects of short-term exposition to $C d$, excess $C u$ ions and jasmonate on oxidative stress appearing in Arabidopsis thaliana. Environmental and Experimental Botany, 2006. 57(1): p. 187-194.

Nourimand, M. and C.D. Todd, Allantoin Increases Cadmium Tolerance in Arabidopsis via Activation of Antioxidant Mechanisms. Plant and Cell Physiology, 2016. 57(12): p. 2485-2496.

Pérez-Chaca, M., et al., Cadmium induces two waves of reactive oxygen species in Glycine max (L.) roots. Plant, cell \& environment, 2014. 37(7): p. 1672.

Sanchez-Zabala, J., C. González-Murua, and D. Marino, Mild ammonium stress increases chlorophyll content in Arabidopsis thaliana. Plant Signaling \& Behavior, 2015. 10(3).
Vidal, E. and R. Gutiérrez, A systems view of nitrogen nutrient and metabolite responses in Arabidopsis. Current opinion in plant biology, 2008. 11(5): p. 521.

Watanabe, S., et al., The purine metabolite allantoin enhances abiotic stress tolerance through synergistic activation of abscisic acid metabolism. Plant, cell \& environment, 2014. 37(4): p. 1022.

Watanabe, S., et al., RNA interference-mediated suppression of xanthine dehydrogenase reveals the role of purine metabolism in drought tolerance in Arabidopsis. FEBS letters, 2010. 584(6): p. 1181.

Werner, A. and C. Witte, The biochemistry of nitrogen mobilization: purine ring catabolism. Trends in plant science, 2011. 16(7): p. 381.

Werner, A., et al., The ureide-degrading reactions of purine ring catabolism employ three amidohydrolases and one aminohydrolase in Arabidopsis, soybean, and rice. Plant physiology, 2013. 163(2): p. 672.

Yang, J. and K. Han, Functional characterization of allantoinase genes from Arabidopsis and a nonureide-type legume black locust. Plant physiology, 2004.134(3): p. 1039. 
Nitrogen Deficiency on Uredide Mediated Stress Resistance (Silzer \& Todd) 